\title{
Like-sign dileptons at the Fermilab Tevatron reexamined in the light of the DESY HERA high- $Q^{2}$ anomaly
}

\author{
Debajyoti Choudhury $^{*}$ and Sreerup Raychaudhuri ${ }^{\dagger}$ \\ Theory Division, CERN, CH 1211 Geneva 23, Switzerland
}

(Received 31 March 1997)

\begin{abstract}
We reexamine like-sign dilepton signals at the Fermilab Tevatron assuming that the excess high- $Q^{2}$ events recently seen at the DESY HERA are due to scalar resonances such as squarks of $R$-parity-violating supersymmetry. For gluinos in the mass range of $200-350 \mathrm{GeV}$, the like-sign dilepton signal can help to make the crucial distinction between the most favored squark explanation and other proposed solutions. [S0556-2821(97)03915-5]
\end{abstract}

PACS number(s): 14.80.Ly, 11.30.Fs, 11.30.Pb

The particle physics community has recently been intrigued by reports of excess high- $Q^{2}$ events in both the H1 [1] and the ZEUS [2] detectors at the DESY ep collider HERA. Concentrated near a parton-momentum fraction of $x \sim 0.5$, these events appear to point to some new effects [3-11] beyond those expected in the standard model (SM). Although the contrary opinion has also been expressed [12], the jury is still out. Even assuming, tentatively, in view of the low statistics, that new physics has indeed been found, opinions differ as to what these effects could be. Various suggestions have been made, including compositeness [3], contact interactions $[11,6]$, and leptoquark [6-10] or squark [4-7] resonances around $200 \mathrm{GeV}$. Of these, the last, namely, squark resonances, constitutes one of the more exciting possibilities, since these events could then be the first experimental signals of supersymmetry (SUSY), a desirable option for various theoretical reasons [13].

The possibility of a squark resonance at HERA was first considered by Hewett [14] and subsequently by others [15]. This obviously requires a violation of $R$ parity [16] in the form of lepton-number-violating $\lambda^{\prime}$ operators [17]. The relevant Lagrangian is given by

$$
\begin{aligned}
\mathcal{L}_{\lambda^{\prime}}= & -\lambda_{i j k}^{\prime}\left[\widetilde{\nu}_{i L} \overline{d_{k R}} d_{j L}+\widetilde{d}_{j L} \overline{d_{k R}} \nu_{i L}+\widetilde{d}_{k R}^{*} \overline{\left(\nu_{i L}\right)^{c}} d_{j L}\right. \\
& \left.-\widetilde{e}_{i L} \overline{d_{k R}} u_{j L}-\widetilde{u}_{j L} \overline{d_{k R}} e_{i L}-d_{k R}^{*} \overline{\left(e_{i L}\right)^{c}} u_{j L}\right]+ \text { H.c. },
\end{aligned}
$$

where the effects of quark mixing have been neglected. In a previous work [4], the present authors pointed out that, if the excess events at HERA are caused by a single dominant $\lambda^{\prime}$ coupling, then the relevant ones are $\lambda_{121}^{\prime}$ (with production of a $\widetilde{c}_{L}$ squark), or $\lambda_{131}^{\prime}$ (with production of a $\widetilde{t}_{L}$ squark). A third possibility has also been suggested [6,5], involving $\lambda_{132}^{\prime}$, with a $\widetilde{t}_{L}$ resonance. A value of $\lambda_{1 i 1}^{\prime}$ in the interval $0.03-0.26$ could give rise to the appropriate signal for the first two options. The third would require $\lambda_{132}^{\prime}$ close to its experimental upper bound of 0.3. Some other operators can

\footnotetext{
*Electronic address: debchou@mail.cern.ch

${ }^{\dagger}$ Electronic address: sreerup@mail.cern.ch
}

contribute, though marginally, for the highest experimentally allowed values of the relevant coupling constant [5].

A crucial feature of the $R$-parity-violating $\left(\mathbb{R}_{p}\right)$ signal is the fact, emphasized by all workers in this field, that the squark can also decay through $R$-parity-conserving channels to charginos and neutralinos, which could give rise to distinctive and unambiguous signals at HERA [18]. Observation of such a signal will be an immediate confirmation of the SUSY hypothesis. We understand that an intensive study of this nature is indeed under way [19]. However, nonobservation of such signals cannot rule out supersymmetry, it will merely tell us that the direct $R$-parity-violating decay of the squark to $e^{+}+d$ has to be the dominant decay channel, while gaugino decay channels are suppressed by large gaugino masses or small couplings. In this case, of course, the decays of the squark will be identical with those of a leptoquark with the same quantum numbers.

An interesting feature of the $R$-parity-violating SUSY solution is that low values of the $\lambda^{\prime}$ coupling in question require large branching ratios to the $R$-parity-violating decay channel $\widetilde{u}_{i L} \rightarrow e^{+}+d$. While this may seem paradoxical at first, we need to recall that the number of excess events seen at HERA essentially scales as $\lambda^{\prime 2} \beta$, where $\beta$ is the branching ratio of $\tilde{u}_{i L}$ to $e^{+}+d$. Thus, small values of $\lambda^{\prime}$ require a large branching ratio and vice versa. Such a scenario is far from unnatural, it can be achieved without difficulty by requiring the charginos and neutralinos to be either heavy or Higgsino-like, which merely corresponds to a restricted range in the parameter space of the minimal supersymmetric standard model (MSSM).

In view of the above, it is now amusing to ask the following question. If, with more statistics from the 1997 run, the high- $Q^{2}$ anomaly does survive as a genuine effect and, moreover, as one compatible with a particle resonance, but no further signals of SUSY are seen at HERA, then the resources of that experiment will have been exhausted insofar as telling the nature of the new physics is concerned. It could be a leptoquark or a squark with a small $(\lessgtr 0.1)$ coupling. In this case, can any other running (or projected) experiment distinguish the supersymmetric option from the others?

The only other running high-energy facility with sufficient energy to produce the "particle" under consideration is the Fermilab Tevatron, running at a center-of-mass energy of 
$1.8 \mathrm{TeV}$. Squarks or leptoquarks can be pair-produced at this machine and their decays would give rise to distinct dilepton + multijet signals. For a squark mass of $200 \mathrm{GeV}$, we estimate the pair production cross section to be around $0.19 \mathrm{pb}$, which is consistent with Ref. [20]. Searches for such processes by the D0 Collaboration have already put a bound of $175 \mathrm{GeV}$ (with $\beta \simeq 1$ ) on the mass of the resonance at $95 \%$ confidence level [21]. This bound is based on a data sample of $117.7 \mathrm{pb}^{-1}$ and may be expected to increase to cover the mass range of interest $(\sim 180-220 \mathrm{GeV})$ as more data accumulate. If these searches fail to show up a resonance, it will be a sign of some serious glitch(es) in our understanding of the HERA anomaly, since most of the new physics solutions, including SUSY, do predict corresponding signals at the Fermilab Tevatron. On the other hand, if the dilepton + jets signal is confirmed, it will inevitably lead us to the question of distinguishability between various theoretical models.

This report addresses the crucial question of distinguishability for the SUSY solution in the context of Fermilab Tevatron searches. An interesting feature of SUSY is that the complete theory requires many more (light?) particles than the squark. One of these is the gluino, the fermionic superpartner of the gluon, which is a strongly interacting Majorana fermion. As a result, relatively large cross sections from QCD production of gluinos are possible and processes involving them can violate fermion number, leading to rather spectacular like-sign dilepton signatures. These have already been studied in some detail in the literature [22-24] and our main purpose is to reexamine their utility in the context of the squarks that can explain the HERA events. The importance of this study lies in the fact that such signals will be present only if the HERA excess is caused by a squark resonance.

We thus need to consider the pair production of gluinos at the Fermilab Tevatron, followed by the decay of each gluino into a quark and a squark, where the squark is the same one that explains the HERA anomaly. Because of the Majorana nature of the gluinos, the squarks coming from their decay have equal probability of forming a like-sign or an unlikesign pair. Subsequent decays of these squarks to $e^{+}+d\left(e^{-}+\bar{d}\right)$ would lead to equal numbers of like-sign and unlike-sign dilepton pairs. This decay chain differs from those studied in Refs. $[22,23]$ in that the squark now has no decays through gaugino-led channels, the latter being the exclusive decay modes studied therein.

Once produced, the gluino will decay into all possible quark-squark pairs that are kinematically allowed. Concentrating on the $\left(\lambda_{121}^{\prime}, \tilde{c}_{L}\right)$ scenario, the most promising one in the context of the HERA events [4-6], the decay channels of interest are

$$
\tilde{g} \rightarrow \bar{c}+\tilde{c}_{L} \rightarrow \bar{c}+e^{+}+d
$$

and

$$
\tilde{g} \rightarrow c+\widetilde{c}_{L}^{*} \rightarrow c+e^{-}+\bar{d},
$$

which lead to a final state with a dilepton pair and jets. The combinations $e^{+} e^{+}, e^{+} e^{-}, e^{-} e^{-}$appear with probability ratio $1: 2: 1$. In other words, if $\tilde{c}_{L}$ were the only light squark, the decay of a gluino pair would lead to a like-sign dilepton final state in exactly one-half the cases. by

Now, the branching ratio of the gluino to $e^{+}+X$ is given

$$
\mathcal{B} \equiv B\left(\tilde{g} \rightarrow e^{+}+X\right)=\frac{\sum_{i} \beta_{i}\left(1+x_{i}-\tilde{x}_{i}\right) \lambda\left(1, x_{i}, \tilde{x}_{i}\right)}{\sum_{i}\left(1+x_{i}-\tilde{x}_{i}\right) \lambda\left(1, x_{i}, \tilde{x}_{i}\right)},
$$

where $x_{i} \equiv m_{q_{i}}^{2} / M_{\tilde{g}}^{2}, \tilde{x}_{i} \equiv m_{\tilde{q}_{i}}^{2} / M_{\tilde{g}}^{2}$, and the sum over $i$ encompasses all squarks for which the decay $\widetilde{g} \rightarrow q_{i} \tilde{q}_{i}$ is kinematically possible. In Eq. (4), $\beta_{i}$ is the branching fraction of the relevant squark to $e^{+}+X$, while $\lambda(a, b, c) \equiv \sqrt{(a-b-c)^{2}-4 b c}$. From our previous discussion, we see that, for $\lambda \lesssim 0.1$,

$$
\beta_{\widetilde{c}_{L}} \simeq 1, \quad \beta_{\widetilde{s}_{L}} \simeq 0, \quad \beta_{\widetilde{d}_{R}} \simeq 0.5 .
$$

As long as $\lambda_{121}^{\prime}$ is the only nonzero $\mathbb{R}_{p}$ coupling, the cascade deacys of the other squarks will result in final states with $e^{ \pm}$or (anti-)neutrinos. Such event topologies have been considered in Refs. [22,23]. Rather than repeat their analyses, which are quite comprehensive for these decay chains, we shall make the conservative assumption that $\beta_{i}=0$ for these squarks. With this simplification, $\mathcal{B}$ in Eq. (4) is determined solely by the gluino and the squark masses. We find that for $\lambda_{121}^{\prime} \simeq 0.1, \mathcal{B}$ is 0.54 or less.

As the masses of the squarks are unknown parameters in the MSSM, we have to make some assumptions for the sake of simplicity. An explanation of the HERA anomaly requires only two squarks to be light. These are the $\widetilde{c}_{L}$ (obviously) and the $\widetilde{s_{L}}$ whose mass is given by the relation

$$
m_{\tilde{s}_{L}}^{2}=m_{\tilde{c}_{L}}^{2}-m_{c}^{2}+m_{s}^{2}-m_{W}^{2} \cos 2 \beta .
$$

Numerically, for $m_{\tilde{c}_{L}} \simeq 200 \mathrm{GeV}$, we find that $m_{\tilde{s}_{L}}$ varies in the range $200-215 \mathrm{GeV}$ as $\tan \beta$ varies from 1 to 50 . The other squarks can be as heavy (or as light) as we please. We make the ad hoc assumption that

$$
m_{\tilde{u}_{R i}}=m_{\tilde{d}_{R i}}=m_{\tilde{u}_{L \alpha}} \equiv m_{\tilde{q}},
$$

where $i=1,2,3$ and $\alpha=1,3$. The masses of $\widetilde{d}_{L}$ and $\widetilde{b}_{L}$ are given by relations analogous to that in Eq. (5). This is not perhaps the optimal spectrum for the signal in question, nor is it the one most favored by model builders, but it is certainly the simplest one. We have checked that the qualitative features of our results do not change with different Ansätze for the squark masses, although the detailed numerics are somewhat affected [25].

Since we want the $\mathbb{R}_{p}$ channel to dominate the squark decay width, the gluino must be heavier than the squark. And so must be the charginos and neutralinos, unless of course the lighter ones are Higgsino dominated. Within the framework of gaugino mass unification, and the CERN $e^{+} e^{-}$collider LEP constraints, this implies a large value for the gaugino mass parameter $M_{2}$. Within the same framework, this would further imply very large gluino masses (generally 
$\gtrsim 500 \mathrm{GeV}$ ) and hence extremely tiny rates at the Tevatron. Note, however, that gaugino mass unification is not very well explored within the context of $R$-parity violation. While grand unification can perhaps be made consistent with $\mathbb{R}_{p}$ [26], such models typically need extra multiplets, all consequences of which are not very well studied. Hence, we shall not assume gaugino-mass unification. The squark mass spectrum does not assume scalar mass unification either. We thus adopt a purely phenomenological approach to the SUSY mass spectrum.

Once we give up gaugino-mass unification as a hypothesis, the soft supersymmetry-breaking parameters $M_{1}, M_{2}, M_{3}$ of the minimal supersymmetric standard model (MSSM) are no longer related. It is then possible to obtain large branching ratios for $\widetilde{c}_{L} \rightarrow e^{+}+d$ for a fairly large range of the parameter space. This is illustrated in the $M_{2}-\mu$ plane for a fixed value $\tan \beta=2$ in Fig. 1. We have chosen two values of the coupling (a) $\lambda_{121}^{\prime}=0.05$ and (b) $\lambda_{121}^{\prime}=0.1$, both of which require a large branching ratio in order to explain the HERA events (see Fig. 2 of Ref. [4]). The region above each contour corresponds to $\beta_{\widetilde{c_{L}}} \geqslant 0.9$. Dashed, solid, and dot-dashed lines correspond to the ratios $M_{1} / M_{2}$ $=2,0.5,0.25$, respectively, of which the second approximates closely to the ratio $M_{1} / M_{2}=\frac{5}{3}\left(g_{1} / g_{2}\right)^{2}$ obtained by assuming gaugino-mass unification purely in the electroweak sector of the MSSM. It is also worth mentioning that for this figure we have included LEP constraints on the $\mathbb{R}_{p}$ scenario in question, these come only from the total width of the $Z$, to which the excess SUSY contribution is [27]

$$
\sum_{i, j=1}^{2} \Gamma\left(Z \rightarrow \tilde{\chi}_{i}^{+} \tilde{\chi}_{j}^{-}\right)+\sum_{i, j=1}^{4} \Gamma\left(Z \rightarrow \tilde{\chi}_{i}^{0} \widetilde{\chi}_{j}^{0}\right) \leq 23.1 \mathrm{MeV}
$$

It is at once apparent that somewhat large values of $M_{2}$ (and $M_{1}$ ) are demanded. Low values of $|\mu|$ are disallowed, partly by the LEP constraint and partly because they correspond to small branching ratios. Finally, we should note that varying $\tan \beta$ does not change the contours much. Accordingly, we have fixed $\tan \beta=2$. Our plots are in good agreement with those of Ref. [6].

The like-sign dilepton signal has been estimated assuming $\beta_{c_{L}}=1$ and using a parton-level Monte Carlo event generator. Our estimates for gluino pair production agree well with those of Ref. [20]. For our simulation, we use the CTEQ3M structure functions [28], which were calculated using the package PDFLIB [29]. We assume a detector rapidity coverage of 3 for both jets and leptons:

$$
|\eta(\ell)|,|\eta(j)|<3 .
$$

All putative jets that lie within a cone of $\Delta R \equiv \sqrt{\Delta \eta^{2}+\Delta \phi^{2}} \leqslant 0.7$ of each other are merged (the momenta added vectorially) to form a single jet. Here, $\Delta \eta$ is the difference of their rapidities and $\Delta \phi$ denotes their azimuthal separation. The major SM backgrounds to the like-sign dilepton signal emanate from $t \bar{t}$ and $W Z$ production [22,23]. These can be severely suppressed by using suitable kinematical cuts. We demand that the leptons have sufficient transverse momentum and be relatively isolated:

$$
p_{T}(\ell)>15 \mathrm{GeV}, \quad E_{T}^{\mathrm{ac}}(\ell)<p_{T}(\ell) / 4,
$$

where $E_{T}^{\mathrm{ac}}(\ell)$ denotes the total hadronic energy within a cone of $\Delta R=0.4$ around the lepton $\ell$ [30]. With the imposition of these cuts, the SM background due to $t \bar{t}$ production was estimated to be as low as $0.3 \mathrm{fb}$, while $2.1 \mathrm{fb}$ comes from $W Z$ production [22]. Clearly, the background level is very low and thus the discovery limit is primarily governed by the signal strength. Our results are consistent with those of Ref. [23], which were checked against an ISAJET-based calculation with reliable cuts and efficiencies.

In Fig. 2, we present contours of cross section for the like-sign dilepton signal in the plane of $m_{\tilde{q}}$, the common squark mass, and $M_{\tilde{g}}$, the gluino mass. We have fixed the mass of the $\widetilde{c}_{L}$ at $200 \mathrm{GeV}$ and $\tan \beta=2$ (which fixes the mass of $\tilde{s_{L}} \simeq 210 \mathrm{GeV}$ ). We have checked that a variation of the former between 180 and $220 \mathrm{GeV}$ and the latter between 1 and 50 makes little visual impact on the figure, although the detailed numbers do change slightly. It should be noted, though, that the lower edges of the curves represent conservative estimates as we have neglected the cascade decays of the squarks into the dilepton channel. Inclusion of these is expected to make the low- $m_{\tilde{q}}$ region more accessible $[22,23]$. Dotted lines in the figure indicate the kinematic reaches of the Tevatron with $0.1 \mathrm{fb}^{-1}$ and $1 \mathrm{fb}^{-1}$ of integrated luminosity. In view of the low background of $\sim 2.4$ $\mathrm{fb}$, we show contours of $5 \mathrm{fb}$ and $50 \mathrm{fb}$, since these imply five events at the Tevatron with, respectively, $0.1 \mathrm{fb}^{-1}$ of data (which are currently available) and with $1 \mathrm{fb}^{-1}$ of data (which should become available with the Main Injector run). The solid contours may, therefore, be taken as discovery limits. The dot-dashed line corresponds to a signal size of 200 $\mathrm{fb}$, which is perhaps rather optimistic. It is immediately obvious that if nature favors us with a somewhat low-lying gluino state $(<350 \mathrm{GeV})$, then it should be possible to observe like-sign dilepton signals at the Tevatron, unless, indeed, there also happens to be a bunch of low-lying squark states around $200 \mathrm{GeV}$ apart from the $\left(\widetilde{c}_{L}, \widetilde{s}_{L}\right)$ pair which have $\beta_{i}=0$ (thereby reducing $\mathcal{B}$ ). On the other hand, if these signals are not seen, it should be possible to rule out a substantial region in the squark-gluino mass plane, which has not been hitherto accessible to Tevatron searches (assuming, of course, that the HERA events are susceptible to a SUSY explanation with a $\tilde{c}_{L}$ resonance).

We now comment on the equally interesting possibility that the HERA events are due to a top squark $\widetilde{t}_{L}$ resonance through a $\lambda_{131}^{\prime}$ (or $\lambda_{132}^{\prime}$ ) coupling. In this case, a strong like-sign dilepton signal requires the gluino to decay to a top-quark-top-squark pair which is only possible when the gluino mass is about $370 \mathrm{GeV}$ or more. This mass range is at the kinematic limit of the Tevatron and, as Fig. 2 makes clear, yields a very small signal. Thus, we conclude that the top-quark-top-squark decay mode of the gluino will not 

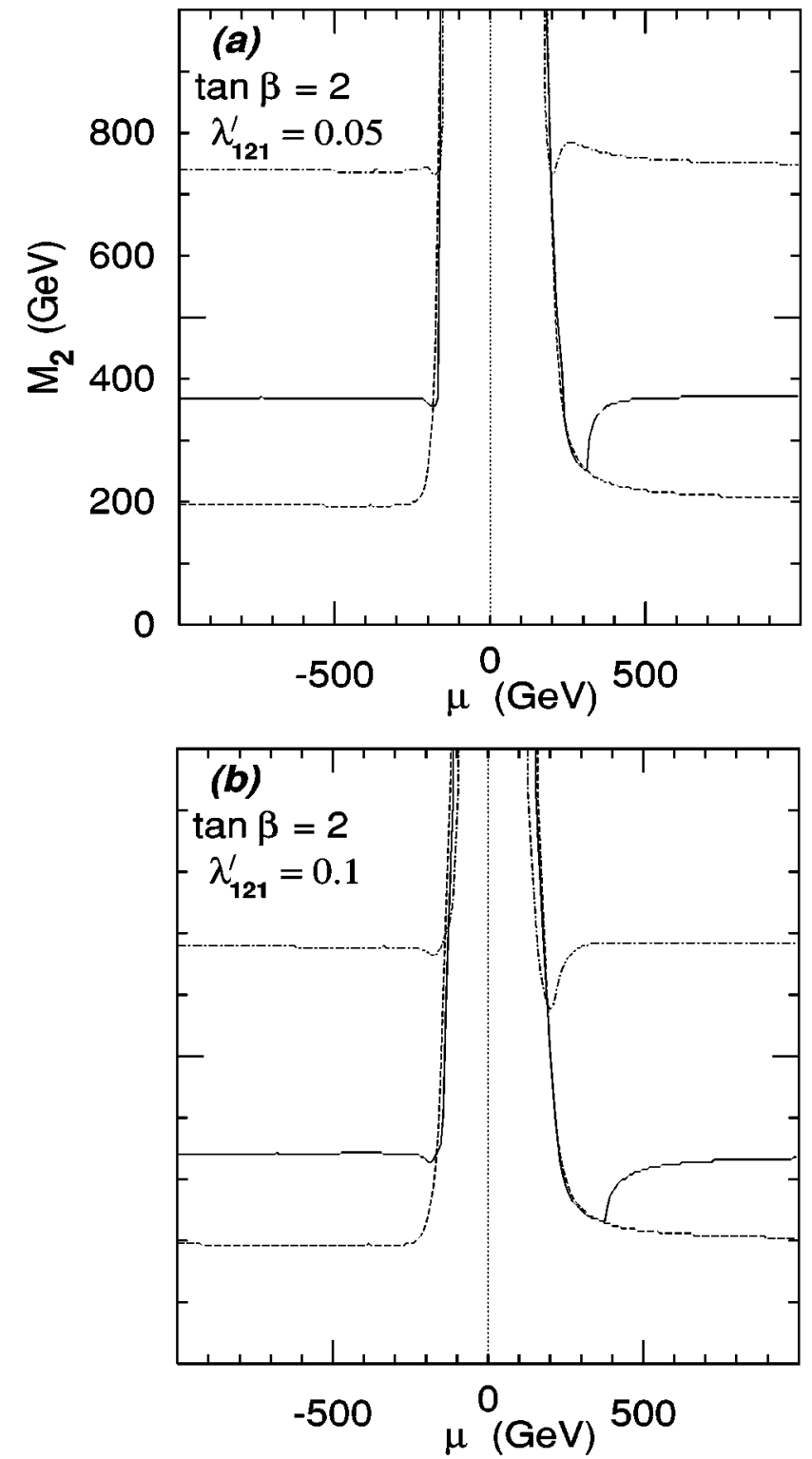

FIG. 1. Contours for $\beta_{\tilde{c}_{L}}=0.9$ in the $M_{2}-\mu$ plane for $\tan \beta=2$ and various values of the ratio $M_{1} / M_{2}$. Dashed, solid, and dotdashed lines correspond to $M_{1} / M_{2}=2,0.5,0.25$, respectively. The dependence on $\tan \beta$ is not very significant.

yield an observable like-sign dilepton signal through the direct $\boldsymbol{R}_{p}$ decay mode. However, the story does not end here, because the presence of an $\sim 200 \mathrm{GeV} \widetilde{t}_{L}$ implies the presence of a light $\widetilde{b}_{L}$. Recalling that the masses are related by

$$
m_{\widetilde{b}_{L}}^{2}=m_{\tilde{t}_{L}}^{2}-m_{t}^{2}+m_{b}^{2}-m_{W}^{2} \cos 2 \beta,
$$

it is easy to check that a $200 \mathrm{GeV} \widetilde{t}_{L}$ implies a $\widetilde{b_{L}}$ in the mass range $105-132 \mathrm{GeV}$. (The lower part of this range is ruled out in the limit of vanishing left-right top squark mixing by constraints from the $\rho$ parameter, although in the presence of mixing this constraint can be relaxed [4].) Unfortunately, even though the gluino can decay dominantly to the

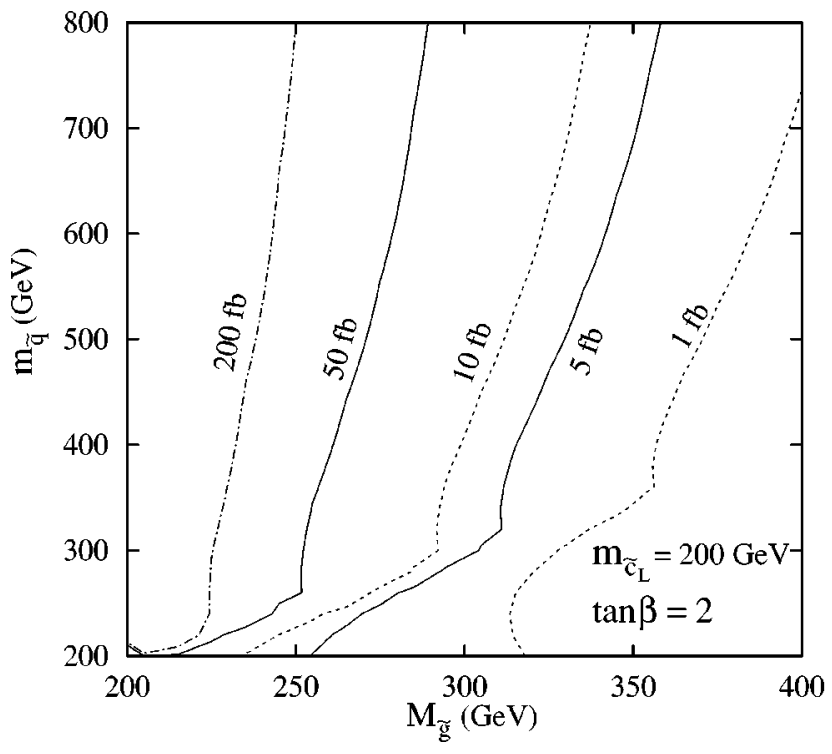

FIG. 2. Contours for the like-sign dilepton cross section at the Tevatron in the $m_{\tilde{g}}-m_{\tilde{q}}$ plane. The dependence on $\tan \beta$ is minimal. Changes in $m_{c_{L}}$ affect only the lower edge of the curve (see text).

$\widetilde{b_{L}}$, the latter (like the $\widetilde{s_{L}}$ ) does not decay to charged leptons and will not contribute to the like-sign dilepton signal. On the other hand, if there is a low-lying $\widetilde{d}_{R}$, one could still get a signal, although even this is suppressed by the fact that $\beta_{\widetilde{d}_{R}} \leqslant 0.5$. We do not go into this aspect any further since there is no compelling reason at the moment to postulate a light $\widetilde{d}_{R}$. Thus we are forced to the conclusion that the likesign dilepton signal is unlikely to be able to distinguish the top squark solution to the HERA anomaly from the possible nonsupersymmetric alternatives. However, a $\widetilde{b_{L}}$ of mass up to $120-130 \mathrm{GeV}$ is likely to be accessible to the thirdgeneration leptoquark search at the Tevatron [21] (in the $b \bar{b}+$ missing $E_{T}$ channel) in the event of a luminosity upgrade. It might also be possible to access a part of the parameter space through cascade decays [22,23].

To summarize, then, we have investigated the possibility of observing like-sign dileptons at the Fermilab Tevatron in view of the possibility, no longer so remote, in view of the HERA anomaly, that there exists a $\widetilde{c_{L}}$ squark of mass around $200 \mathrm{GeV}$. If there is also a gluino in the $200-350 \mathrm{GeV}$ range, it might be possible to see such signals at the Tevatron, if not now, then in the near future, with the projected luminosity upgrade. Even with the present data sample it should be possible to explore a hitherto untouched part of the parameter space, at least for the scenario in question. Unfortunately, the alternative SUSY solution of the HERA anomaly with a 200 $\mathrm{GeV} \widetilde{t}_{L}$ cannot be addressed well through this particular signal. Even so, much can be achieved by searching for likesign dileptons in the present data sample and we would urge upon our experimental colleagues the importance of carrying out such searches with the highest priority.

The authors would like to thank Manuel Drees and N. K. Mondal (D0 Collaboration) for discussions. 
[1] H1 Collaboration, C. Adloff et al., DESY Report No. 97-024.

[2] ZEUS Collaboration, J. Breitweg et al., DESY Report No. 97025 .

[3] S. L. Adler, Princeton Report No. IASSNS-HEP-97-12, hep-ph/9702378 (unpublished).

[4] D. Choudhury and S. Raychaudhuri, Phys. Lett. B 401, 54 (1997).

[5] H. Dreiner and P. Morawitz, Report No. hep-ph/9703279 (unpublished).

[6] G. Altarelli, J. Ellis, G. F. Giudice, S. Lola, and M. L. Mangano, CERN Report No. CERN-TH-97-040, hep-ph/9703276 (unpublished).

[7] J. Kalinowski, R. Rückl, H. Spiesberger, and P. M. Zerwas, DESY Report No. 97-038, hep-ph/9703288 (unpublished).

[8] J. Blümlein, DESY-Zeuthen Report No. 97-032, hep-ph/9703287 (unpublished).

[9] K. S. Babu, C. Kolda, J. March-Russell, and F. Wilczek, Princeton Report No. IASSNS-HEP-97-04, hep-ph/9703299 (unpublished).

[10] J. L. Hewett and T. G. Rizzo, SLAC Report No. SLAC-PUB7430, hep-ph/9703337 (unpublished).

[11] V. Barger, K. Cheung, K. Hagiwara, and D. Zeppenfeld, Madison Report No. MADPH-97-991, hep-ph/9703311 (unpublished).

[12] M. Drees, Asia Pacific Center Report No. APCTP 97-03, hep-ph/9703332 (unpublished).

[13] H. P. Nilles, Phys. Rep. 110, 1 (1984); P. Nath, R. Arnowitt, and A. H. Chamseddine, Applied $N=1$ Supergravity (World Scientific, Singapore, 1984); H. E. Haber and G. L. Kane, Phys. Rep. 117, 75 (1985).

[14] J. Hewett, in Snowmass Summer Study, edited by E. L. Berger (World Scientific, Singapore, 1990), p. 566.

[15] T. Kon and T. Kobayashi, Phys. Lett. B 270, 81 (1991); T. Kon, T. Kobayashi, and K. Nakamura, in Physics at HERA, Hamburg, 1991, Vol. 2, p. 1088; J. Butterworth and R. Dreiner, Nucl. Phys. B397, 3 (1993); T. Kon, T. Kobayashi, S. Kitamura, K. Nakamura, and S. Adachi, Z. Phys. C 61, 239 (1994); T. Kon, T. Kobayashi, and S. Kitamura, Phys. Lett. B 333, 263 (1994); T. Kobayashi, S. Kitamura, and T. Kon, Int.
J. Mod. Phys. A 11, 1875 (1996); E. Perez, Y. Sirois, and H. Dreiner, in Future Physics at HERA, edited by G. Ingelman, A. De Roeck and R. Klasner (DESY, Hamburg, 1996), p. 295.

[16] G. Farrar and P. Fayet, Phys. Lett. 76B, 575 (1978).

[17] S. Weinberg, Phys. Rev. D 26, 287 (1982); N. Sakai and T. Yanagida, Nucl. Phys. B197, 533 (1982); C. S. Aulakh and R. Mohapatra, Phys. Lett. 119B, 136 (1982).

[18] See, e.g., H1 Collaboration, S. Aid et al., Z. Phys. C 71, 211 (1996).

[19] Y. Sirois, talk presented on behalf of the H1 Collaboration at CERN, 1997.

[20] W. Beenakker, R. Höpker, M. Spira, and P. M. Zerwas, DESY Report No. DESY 96-150, hep-ph/9610490 (unpublished).

[21] D0 Collaboration, J. A. Wightman, Les Rencontres de Physique de la Vallée d'Aoste, La Thuile, 1997.

[22] H. Baer, C. Kao, and X. Tata, Phys. Rev. D 51, 2180 (1995).

[23] M. Guchait and D. P. Roy, Phys. Rev. D 54, 3276 (1996).

[24] H. Dreiner, M. Guchait, and D. P. Roy, Phys. Rev. D 49, 3270 (1994).

[25] One might argue that the $\widetilde{d}_{R}$ mass should be close to the $\left(\widetilde{c_{L}}, \widetilde{s_{L}}\right)$ pair as it also participates in the same $\boldsymbol{R}_{p}$ interaction. Obviously, this is not compelling. Further, it is easy to see that this will not affect our numerical results unless one considers the $\left(\widetilde{t}_{L}, \widetilde{b}_{L}\right)$ case.

[26] K. Tamvakis, Phys. Lett. B 382, 251 (1996); 383, 307 (1996); R. Hempfling, Nucl. Phys. B478, 3 (1996), and references therein.

[27] The LEP Electroweak Working Group, CERN Report No. LEPEWWG/96-28, 1996 (unpublished).

[28] CTEQ Collaboration, R. Brock et al., Rev. Mod. Phys. 67, 157 (1995); CTEQ Collaboration, J. Botts et al., Phys. Lett. B 304, 159 (1993).

[29] H. Plothow-Besch, Comput. Phys. Commun. 75, 396 (1993).

[30] Our cuts are identical to those of Ref. [22]. Reference [23], on the other hand, uses a slightly different set: $E_{T}^{\mathrm{ac}}(\ell)<5 \mathrm{GeV}$ and $\mid \eta /<1$. While our analysis is not very sensitive to the difference in the isolation cut, the reduced rapidity range will moderately degrade the results. 